\title{
ECDSA: The Virtual Signature Set of Rules of a Higher Internet
}

\author{
Dr. Kanakam Siva Rama Prasad M.Tech $(\mathrm{CSE})$,
}

Ph. D, Pace Institute of Technology and sciences, Ongole, Andhra Pradesh,

\begin{abstract}
Computerized Currency is an electronic kind of cash. These days, everything is developing into digitization measure. This contains all properties like actual cash and furthermore permits prompt trades which will be reliably executed across the world while partner with upheld contraptions and organizations. In this paper we presented the SHA3-512 bit hashing algorithm and ECDSA algorithm for generation of digital signature. The Elliptic curve cryptography (ECC) is one of the greater promising technology on this area. ECC-enabled TLS is quicker and greater scalable on our servers and presents the equal or higher protection than the default cryptography in use at the web. one of the elliptic curve algorithm, the elliptic curve virtual signature algorithm (ECDSA), may be used to enhance overall performance at the Internet. CloudFlare now helps custom ECDSA certificate for our clients and that's true for all people the use of the Internet.
\end{abstract}

Keywords: Crypto currencies, Hasing, SHA3-512, ECDSA.

\section{Introduction}

Advanced Currency is an electronic kind of cash. These days, everything is advancing into digitization measure. This contains all properties like actual cash and furthermore permits quick trades which will be reliably executed across the world while partner with upheld contraptions and organizations. There are various kinds of Digital monetary forms like Cryptocurrencies, virtual monetary standards, monetary organization advanced monetary standards, and e-Cash and so forth are the examples of computerized money. One truth is that everyone cryptographic forms of money are computerized monetary standards, yet not all advanced monetary forms are crypto. Computerized cash allows an overall trade of proprietorship additionally as momentary showcasing and might be used to search for products and administrations. With this computerized monetary standards enjoy various benefits as observes a lower cost, installments between the executing parties without the need for any go-betweens, Digital money based electronic trades enjoying benefits like misrepresentation insurance, easier worldwide installments, and so on There are for the most part two kinds of Digital Currency are there Virtual Currency and
Cryptocurrency. Virtual Currency-Virtual cash might be a computerized money that is used inside a particular planned organization. Cryptographic money it's a computerized cash that has genuine worth, as Bitcoin. This sort of cutting edge cash relies upon mathematical estimations with tokens being moved electronically over the online through disseminated frameworks organization. Cryptographic money isn't coordinated with the economy of any country.

\section{Cryptocurrency}

Cryptocurrency makes it less complicated to steer global exchanges. A cryptocurrency is a virtual foreign money secured through cryptography and primarily based totally on blockchain generation. A blockchain is a digital ledger that organizes facts into gadgets or organizations which can be known as blocks, wherein every block containing a group of facts and having their very own precise garage capability wherein as soon as filled, are chained onto the preceding block, developing a facts chain referred to as a "blockchain." All new data added after a newly inserted block is compiled into a brand new block and introduced to the chain. Blockchain Technologies utilized by maximum cryptocurrencies are unfold throughout many servers, making cryptocurrency decentralization is the distinguishing component that separates cryptocurrency from different asset classes. The cryptocurrency has transparency 
because because of this blockchain generation nobody is constrained having a have a take an observe the transaction facts at any time. Bitcoin is on the market all around the international everywhere in the clock which makes it a whole lot greater accessible. Most of the time, buyers can get admission to cryptocurrencies with cell telephones in a hassle-loose manner. The blockchain database is made of a sequence of difficult-to-clear up cryptographic puzzles. Cryptocurrency transfers are greater dependable than normal digital transactions. Cryptocurrencies are inflation-covered in maximum of the cases. There are such a lot of cons of cryptocurrencies. Like unlawful activities, volatility, facts loss, hacking etc.. The following are the pinnacle biggest cryptocurrencies - Bitcoin (BTC) Satoshi Nakamoto, an alias, created in 2009 is the most important cryptocurrency in with marketplace capitalization of greater than $\$ 1$ trillion, as stated through CoinMarketCap, Currently, there are greater than 18.five million Bitcoin tokens which are in movement across the international. Approximately 900 new bitcoins are mined daily. Ethereum (ETH) become created in 2015 with the number one consciousness on decentralized applications. The call of the token utilized in Ethereum is Ether. Accroding to the facts supplied through CoinMarketCap,

It has a marketplace capitalization of greater than $\$ 300$ million. Bitcoin Cash (BCH) become added in 2017 to enhance the capabilities of bitcoin. Today, it's far one of the maximum famous cryptocurrencies. It has a block length of $8 \mathrm{MB}$ compared to a block length of $1 \mathrm{MB}$ in Bitcoin and, therefore, is a quicker opportunity to Bitcoin. It has a marketplace capitalization of about \$sixteen million. Litecoin (LTC)-Charlie Lee created Litecoin in 2011. This become added to gain quicker transactions, decrease fees, and greater focused miners. Some greater famous cryptocurrenices are Binance Coin, Tether (USDT), Dogecoin (DOGE), Uniswap (UNI). With the boom withinside the hobby in cryptocurrencies, there was a speedy increase withinside the wide variety of latest cryptocurrencies getting into the crypto-area withinside the beyond few years and in step with quite a few crypto-researchers, this upward thrust is anticipated to continue. Cryptocurrency exchanges are the ones which facilitate the buying and selling of cryptocurrencies are shape a subset of virtual foreign money exchanges. They acts as interface among transactions among cryptocurrencies and different virtual currencies. Cryptocurrency exchanges both centralized or decentralized. Centralized cryptocurrency exchanges function middlemen among customers and sellers. The idea is just like the concept of inventory exchanges. Since they may be run and owned through a corporation, they may be greater dependable and nearly all the crypto trades take vicinity in centralized exchanges. Eg. Coinbase, GDAX, Kraken, and Gemini. Decentralized cryptocurrency exchanges do now no longer have any middlemen and permit bilateral transactions. They are greater steady than centralized cryptocurrency exchanges which can be vulnerable to hacks however are riskier because of the absence of any intermediary. Eg: AirSwap, io, Barterdex, and Blocknet.

An digital coin as a sequence of virtual signatures. These virtual signatures confer realistic manage, and in maximum cases, possession over the cash held in any given script, and may be used as a file of custodial manage to hint transfers of manage lower back thru the records of the ledger. The Bitcoin ledger is a file of all legitimate transactions which have ever been transmitted to the network. The ledger is shaped as a Directed Acyclic Graph (DAG) wherein every transaction is a node. The graph begins offevolved on the Coinbase transaction of the primary block ever determined and through chains of virtual signatures maps out the complete records of legitimate alternate actions, permitting the tracing of all bitcoins lower back to their creation. In the below Fig. 1 the Bitcoin is transferred one to another by applying the hash function with signature generation algorithm. In this paper we used the sha3:512 algorithm is used to get the hash value

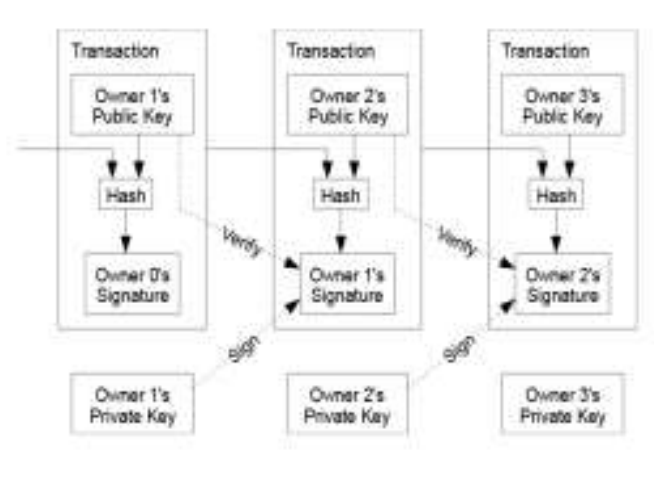

Figure-1: Transfer of Bitcoin from one to another 


\section{Hash Value Generation Algorithms}

With a clean concept concerning the importance of hashing in blockchain, it's far crucial to recognise approximately hashing algorithms. The steady hashing set of rules or SHA is the maximum not unusualplace hash feature encouraged via way of means of the National Institute of Standards and Technology (NIST). The brilliant successors of SHA which includes SHA-1, SHA-2, and SHA-three have won profound popularity for his or her capabilities. Let us check their details. SHA-1 ought to take enter of nearly any period after which generate a 160bit message along processing messages in blocks of 512-bit size. If message period isn't always a more than one of 512-bit, then the SHA set of rules ought to pad up the message with statistics in order that it may attain the subsequent closest more than one of 512-bit. SHA-2 is currently one of the favourite algorithms withinside the cryptography community, despite the fact that with sure setbacks like withinside the SHA-1 set of rules. After its creation in 2001, SHA-2 has been via widespread adjustments over time with the appearance of 4 editions. The 4 extraordinary editions encompass SHA-256, SHA-224, SHA512, and SHA-384, with SHA-256 being a broadly followed cryptographic set of rules. SHA256 can create a 256-bit message digest via the usage of 512-bit block size, even as SHA-224 makes use of a truncated model of SHA-256 for growing a 224-bit message digest the use of the 512-bit block size. SHA-512 ought to create a 512-bit message digest via way of means of the use of the 1024-bit block size, and SHA-384 makes use of a truncated model of SHA-512. SHA-384 can generate a 384-bit message digest via way of means of leveraging a 1024-bit block size. The SHA-three algorithms are the modern day additions in steady hashing algorithms displaying the significance of hashing in blockchain. SHA-three got here into lifestyles in 2015 and fall at the equal traces as MD5 set of rules standards. It has the functionality to function an alternative for SHA-2 even as additionally supplying comparable editions and hash lengths. The most effective distinction of SHA-three is that it offers opportunities of higher security. A digital signature isn't merely a message signed using a given keypair, but is a link to an identity. The European Union legislation on digital signatures states that signatures correspond to "data in electronic form which are attached to or logically associated with other electronic data and which serve as a method of authentication". Bitcoin script allows users to lock/unlock their bitcoin in different ways. In the above diagram we given the string is "This is Siva Rama Prasad" by using the 64 bit hash function "sha3-512" i.e. "Secure Hash Algorithm 3 of 512 bit" produces the following hash value

13FAA42E86B4183960762250951FE5274EE769 9E33A61BF04489FE216A3706C54724C64F783 FB49715F096799C3BFA3FE2BOF176F0DDA27 2872F4B53CF6EC837

By submitting resultant hash value to the Elliptical Curve Digital Signature Algorithm we will get the signature as the following output this process repeats for every new transfer.

\section{Elliptical Curve Digital Signature Algorithm (ECDSA)}

Cryptocurrency makes it easier to steer international exchanges. A cryptocurrency is a virtual forex secured with the aid of using cryptography and primarily based totally on blockchain technology. A blockchain is a digital ledger that organizes records into devices or businesses that are referred to as blocks, in which every block containing a group of records and having their very own precise garage capability in which as soon as filled, are chained onto the preceding block, developing a records chain called a "blockchain." All new records added after a newly inserted block is compiled into a brand new block and delivered to the chain. Blockchain Technologies utilized by maximum cryptocurrencies are unfold throughout many servers, making cryptocur $\neg$ This set of rules is the elliptic curve analogue of the DSA set of rules. This changed into first proposed withinside the 12 months 1992 with the aid of using the Scott Vanstone. At gift The Accredited Standards Committee X9 Inc. (X9) introduced that it has posted a brand new fashionable version X9.142, The Elliptic Curve Digital Signature Algorithm (ECDSA). This fashionable defines a mechanism to facilitate the steady authentication and nonrepudiation of records in economic and different on-line transactions, the usage of the ECDSA. X9.142 specifies a virtual signature set of rules, the ECDSA. Elliptic curve signatures, which includes ECDSA, are the quickest and smallest to be had steady virtual signatures, they may be now used widely, frequently changing legacy RSA signatures. Some of the maximum distinguished web sites at the moment are secured with the 
assist of ECDSA. The net server generates an ECDSA signature in a steady handshake for every net visitor. Web browsers confirm the ECDSA signature, displaying this verification to the person with the aid of using manner of a steady lock icon subsequent to the net address. A blockchain economic transaction may additionally use an ECDSA signature to completely bind the transaction on a ledger. The X9.142 ECDSA fashionable presents techniques and standards for producing the private and non-private keys that the ECDSA requires, and procedural controls wished for steady use of the set of rules with those keys. The fashionable additionally presents techniques and standards for producing the elliptic curve area parameters that the ECDSA requires, and procedural controls for steady use of the set of rules with those area parameters. It replaces X9.62-2005, an in advance specification of the ECDSA. As shown in the figure-2, this paper exploring the generation of Digital signature Using ECDSA and making the verification of the signature at the receiver end.

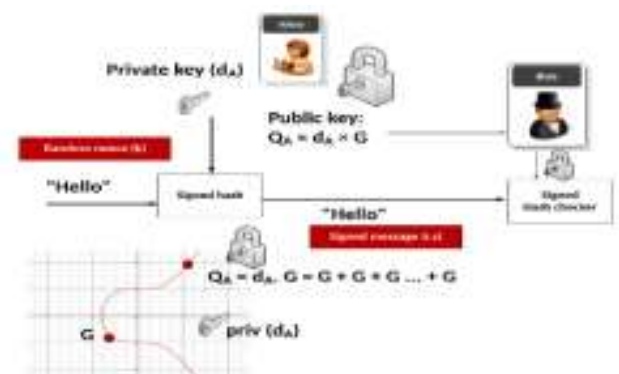

Figure-2:

Generation and verification process of Digital signature

Alice has a private key $\left(d_{A}\right)$ and a public key $\left(Q_{A}=d_{A} \cdot G\right)$. She then signs a message $(M)$ with the following:

1. Create a hash of the message $\mathrm{e}=\mathrm{HASH}$ (m).

2. Let $h$ be the Ln be the leftmost bits of e, Ln has a bit length of the group order $\mathrm{N}$.

3. Create a random number $\mathrm{k}$ which is between 1 and $\mathrm{N}-1$.

4. Calculate a point on the curve as $(\mathrm{x} 1, \mathrm{y} 1)=$ $\mathrm{k} \times \mathrm{G}$.

5. Calculate $r=x 1(\bmod N)$. If $r=0$, go back to Step 3.

6. Calculate $\mathrm{s}=\mathrm{k}^{-1}\left(\mathrm{~h}+\mathrm{rd}_{\mathrm{A}}\right)(\bmod \mathrm{N})$ If $\mathrm{s}=0$, go back to Step 3 .

7. The signature is the pair $(r, s)$.
Bob will verify with following algorithm:

1. Create a hash of the message e $=\mathrm{HASH}(\mathrm{m})$.

2. Let $h$ be the Ln leftmost bits of e.

3. Calculate $\mathrm{c}=\mathrm{s}-1(\bmod \mathrm{N})$

4. Calculate $\mathrm{u} 1=\mathrm{h} \cdot \mathrm{c}(\bmod \mathrm{N})$ and $\mathrm{u} 2=\mathrm{r} \cdot \mathrm{c}$ $(\bmod N)$.

5. Calculate the curve point $(\mathrm{x} 1, \mathrm{y} 1)=$ $\mathrm{u} 1 \times \mathrm{G}+\mathrm{u} 2 \times \mathrm{QA}$. If $(\mathrm{x} 1, \mathrm{y} 1)=\mathrm{O}$ then the signature is invalid.

6. The signature is valid if $\mathrm{r}=\mathrm{x} 1(\bmod n)$, invalid otherwise.

7.

\section{Python Implementation of the ECDSA algorithm}

from ecdsa import

SigningKey,NIST192p,NIST224p,NIST256p,NIS

T384p,NIST521p,SECP256k1

import base64

import sys

import binascii

msg="Hi welcome"

type $=1$

cur $=$ NIST192p

if (len(sys.argv) $>1)$ :

$\mathrm{msg}=\mathrm{str}(\operatorname{sys} . \operatorname{argv}[1])$

if (len(sys.argv)>2):

type $=$ int $(\operatorname{sys} . \operatorname{argv}[2])$

sk $=$ SigningKey.generate $($ curve $=$ cur $)$

vk = sk.get_verifying_key()

print("Secret

key:|t",binascii.hexlify(sk.to_string()))

print("Public

key:lt",binascii.hexlify(vk.to_string()))

signature $=\operatorname{sk} \cdot \operatorname{sign}(\operatorname{msg} . \operatorname{encode}())$

print("Message: $\mid t ", m s g)$

print("Type:ltlt",cur.name)

print ("=========================")

print("Signature:It",base64.b64encode(signature)) print("Signature:It",binascii.hexlify(signature).dec ode())

$\operatorname{print}("======================")$

print("Signatures match:lt",vk.verify(signature, msg.encode()))

\section{Results}


Secret key:

b'5a15138b8918f694019b979a9ed8b9d777a6a4fe a7a2938f'

Public key:

b'cedfa558314934f136e3449a466cb3fc70e138265

4e508930454d627b00044dd1bd2f6506371e150fc 55775190d8f552'

Message: Hello

Type: NIST192p

Signature:

b'qWX3N/yYARV46zBn/jR3UfznrI4EFW3/JmA HP7RR0ubH7MdI8RkRtg2E3nTpxFGH'

Signature: a965f737fc98011578eb3067fe347751fce7ac8e04 156dff2660073fb451d2e6c7ecc748f11911b60d84 de $74 \mathrm{e} 9 \mathrm{c} 45187$

Signatures match: True

\section{Conclusion}

Elliptic curve cryptography is an effective era which could permit quicker and extra stable cryptography throughout the Internet. The time has come for ECDSA to be broadly through allowing clients to apply ECDSA certificate on their CloudFlare-enabled sites deployed at the web. We are taking the primary steps toward that intentions

\section{References:}

1. International Journal of Economics \& Finance Research \& Applications Vol. 2, Issue 2 - 2018 (C) Eureka Journals 2018. All Rights Reserved. ISSN: 2581-4249 Using "Crypto Currency And Associated Advantages And Disadvantages" Rashmi Priya Sharma*, Arabinda Sharma

2. "An Analysis of Cryptocurrency, Bitcoin, and the Future" Peter D. DeVries Professor of MIS University of Houston - Downtown One Main Street, FAMIS Department, B428, Houston, TX 77002 United States of America- International Journal of Business Management and Commerce Vol. 1 No. 2; September 2016.

3. S. M. Farooq, S. M. Suhail Hussain and T. S. Ustun, "Elliptic Curve Digital Signature Algorithm (ECDSA) Certificate Based Authentication Scheme for Advanced Metering Infrastructure," 2019 Innovations in Power and Advanced Computing Technologies (i-PACT), 2019, pp. 1-6, doi: 10.1109/i-PACT44901.2019.8959967.
4. International Journal of Computer Applications (0975 - 8887) Volume 2 - No.2, May 201021 Implementation of Elliptic Curve Digital Signature Algorithm Aqeel Khalique Kuldip Singh Sandeep Sood Department of Electronics \& Computer Engineering, Indian Institute of Technology Roorkee Roorkee, India

5. "Efficient and Secure ECDSA Algorithm and its Applications: A Survey" Mishall AlZubaidie1,2, Zhongwei Zhang2 and Ji Zhang2 1Thi-Qar University, Nasiriya 64001, Iraq 2 Faculty of Health, Engineering and Sciences, University of Southern Queensland, Toowoomba, QLD 4350, Australia 